\title{
Unilateral Diaphragm Paralysis Associated With Neurosyphilis: A Case Report
}

\author{
Sungchul Huh, $\mathrm{MD}^{1}$, Jae Heun Chung, $\mathrm{MD}^{2}$, Han Jo Kwon, $\mathrm{MD}^{3}$, Hyun-Yoon Ko, MD, PhD ${ }^{1}$ \\ Departments of ${ }^{1}$ Rehabilitation Medicine, ${ }^{2}$ Pulmonology, Allergy and Critical Care Medicine, and ${ }^{3}$ Ophthalmology, \\ Pusan National University Yangsan Hospital, Pusan National University School of Medicine, Yangsan, Korea
}

\begin{abstract}
Diaphragm dysfunction can originate from various etiologies, and bilaterality of the dysfunction depends on the cause. Symptoms of diaphragm dysfunction vary depending on the degree of phrenic nerve denervation, spinal cord lesion, and involvement of the diaphragm. Several infectious diaphragmatic dysfunctions have been reported, including the human immunodeficiency virus, poliovirus, West Nile virus, and dengue virus. Here, we report a case of unilateral diaphragm paralysis in a 34-year-old man with neurosyphilis.
\end{abstract}

Keywords Neurosyphilis, Diaphragm, Phrenic nerve

\section{INTRODUCTION}

There are many causes of diaphragm paralysis. Several infectious agents causing diaphragmatic dysfunctions have been reported, including the human immunodeficiency virus (HIV), poliovirus, West Nile virus, and dengue virus [1-3]. Syphilis is a sexually transmitted disease caused by Treponema pallidum and occasionally affects the central nervous system as neurosyphilis. Previous studies have reported that the clinical features of neurosyphilis include meningitis, ocular syphilis, stroke, spinal cord disease, and seizures. Since the clinical manifestations of neurosyphilis vary, clinicians often experience difficulties when diagnosing this disease. However, involvement of the peripheral nerves, including the phren- ic nerve, is very rare and has only been reported in a few cases of syphilis [4-7]. Although diaphragm paralysis is rarely observed in neurosyphilis, it may be critical if present.

In the present case, plain chest X-ray, ultrasonography, and nerve conduction studies of the phrenic nerve with needle electromyography of the diaphragm were performed, and unilateral phrenic nerve palsy was diagnosed as a result. In addition, the laboratory tests, cerebrospinal fluid (CSF) analysis, and ophthalmologic evaluation confirmed the presence of neurosyphilis, which manifested as optic neuropathy.

Received December 17, 2019; Revised March 18, 2020; Accepted April 20, 2020; Published online July 28, 2020 Corresponding author: Hyun-Yoon Ko

Department of Rehabilitation Medicine, Pusan National University Yangsan Hospital, Pusan National University School of Medicine, 20 Geumo-ro, Mulgeum, Yangsan 50612, Korea. Tel: +82-55-360-4000, Fax: +82-55-360-4251, E-mail: drkohy@gmail.com

ORCID: Sungchul Huh (http://orcid.org/0000-0002-7740-8050); Jae Heun Chung (http://orcid.org/0000-0003-4519-4219); Han Jo Kwon (http://orcid. org/0000-0003-2973-9725); Hyun-Yoon Ko (http://orcid.org/0000-0001-5506-7219).

@ This is an open-access article distributed under the terms of the Creative Commons Attribution Non-Commercial License (http://creativecommons.org/ licenses/by-nc/4.0) which permits unrestricted noncommercial use, distribution, and reproduction in any medium, provided the original work is properly cited. Copyright $\odot 2020$ by Korean Academy of Rehabilitation Medicine 


\section{CASE REPORT}

A 34-year-old man experienced sore throat and penile ulcers for 3 months and complained of no symptoms associated with upper respiratory infections, such as coughing or sputum. He reported having sexual intercourse approximately 3 months previously. The Venereal Disease Research Laboratory titer was 1:128. His serum rapid plasma reagin titer was $>1: 64$, and the fluorescent treponemal antibody absorbed (FTA-ABS) test was positive. He was diagnosed with syphilis based on these laboratory test results; however, other tests for HIV and hepatitis $\mathrm{B}$ and $\mathrm{C}$ were negative.

The patient also complained of blurred vision. Based on the ophthalmologic manifestations and positive serologic tests, we suspected the presence of neurosyphilis. Fundus examination showed optic neuropathy of the left eye, and CSF analysis revealed lymphocyte-dominant pleocytosis (19 white blood cells/ $\mu \mathrm{L}$ with $84 \%$ lymphocytes) with reactive CSF FTA-ABS, which indicated the presence of neurosyphilis. Ceftriaxone $2 \mathrm{~g}$ was administered intravenously once daily for 14 days. We started $60 \mathrm{mg}$ prednisolone once daily with antibiotics to preserve the visual acuity. Prednisolone $60 \mathrm{mg}$ was administered for 1 week and then tapered by $10 \mathrm{mg}$ weekly. The patient's blurred vision normalized within a month.

The chest X-ray initially showed marked elevation of the right diaphragm to the 7 th intercostal space, which was continued in follow-up serial images. The right diaphragm, however, had been normal in images taken 1 year before. These posteroanterior chest images were acquired in the upright posture. The patient had no history of trauma that could have caused abnormal diaphragmatic movements. Chest and abdominal computed tomography revealed no space-occupying lesions along the course of the right phrenic nerve. Diaphragm ultrasonography revealed right diaphragm paralysis with no actual excursion in the M-mode or thickness change in the B-mode (Fig. 1). Ultrasonography revealed that the left diaphragmatic excursion was $>7 \mathrm{~cm}$ during forceful breathing. The thickness of the left diaphragm was 0.38 $\mathrm{cm}$ at end-expiration and $0.58 \mathrm{~cm}$ at end-inspiration, while that of the right diaphragm was $0.22 \mathrm{~cm}$ and did not change during breathing.

Electrophysiological studies revealed that the amplitude of the compound muscle action potential of the right phrenic nerve had severely decreased $(0.1 \mathrm{mV})$, while that of the left phrenic nerve was $0.7 \mathrm{mV}$ (Fig. 2). Needle electromyography of the right diaphragm showed an abnormal spontaneous activity of positive sharp waves and fibrillation potentials and no recruitment of motor units during inspiration. These findings provided the evidence of a lesion associated with the right phrenic nerve. The pulmonary function test revealed a forced vital capacity (FVC) of $2.10 \mathrm{~L}$ or $50 \%$ of the predicted value, forced expiratory volume in 1 second $\left(\mathrm{FEV}_{1}\right)$ of $1.62 \mathrm{~L}$ or $46 \%$ of the predicted value, and $\mathrm{FEV}_{1} / \mathrm{FVC}$ ratio of $77.1 \%$.

We initially excluded multiple sclerosis, spinal cord lesions, and motor neuron disease as potential diagnoses because there were no apparent neurological motor weaknesses, sensory disturbances, or segmental reflex abnormalities in the upper or lower extremity. There
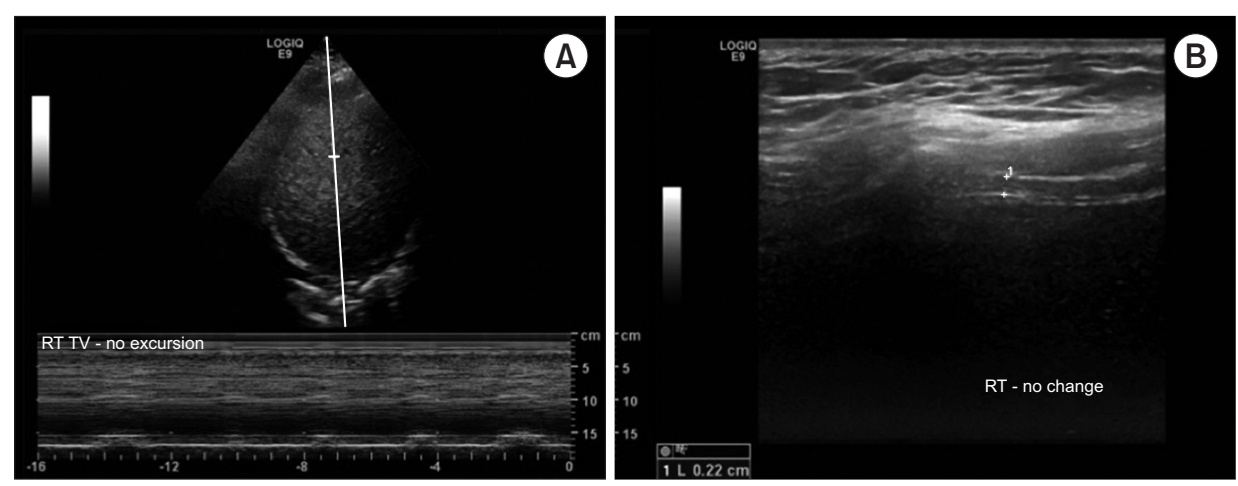

Fig. 1. Initial ultrasonography of the right diaphragm. Ultrasonography was performed in two different views. The hyperechoic right diaphragm in the 7th intercostal space was identified at the distal end of the liver, which served as an acoustic window. (A) The excursion of the diaphragm was viewed through M-mode and there was no movement of the right diaphragm. (B) The thickness of the right diaphragm is viewed through B-mode and the value revealed $0.22 \mathrm{~cm}$. 

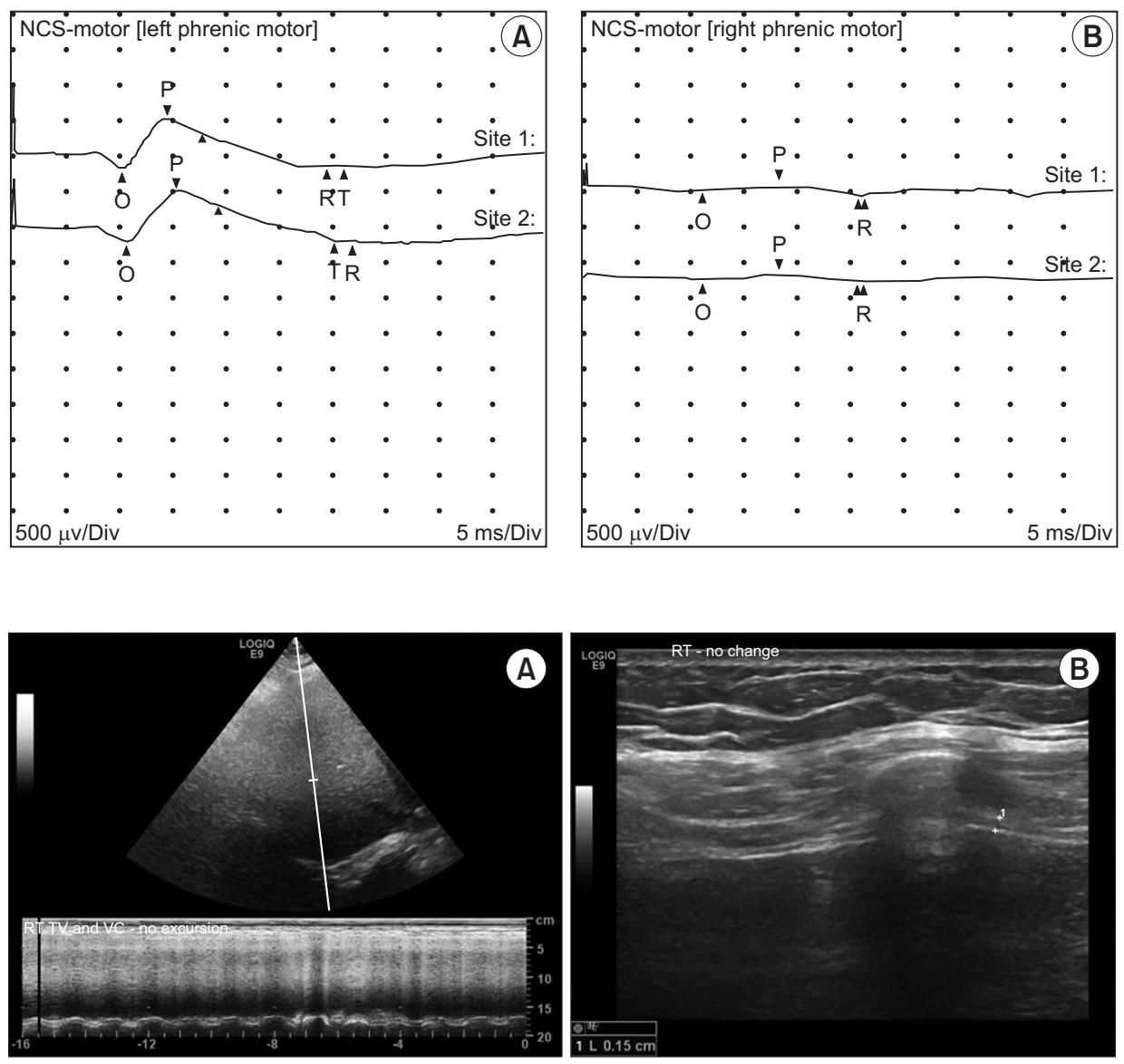

Fig. 2. Nerve conduction studies of (A) right and (B) left phrenic nerves. The compound muscle action potential of the right phrenic nerve showed a decreased amplitude $(0.1 \mathrm{mV})$, while the left showed a normal amplitude $(0.7$ $\mathrm{mV})$.

Fig. 3. Follow-up ultrasonography of the right diaphragm. (A) The follow-up ultrasonography after 2 months showed a paradoxical movement of the right diaphragm, which indicated complete paralysis. (B) The thickness of the right diaphragm revealed $0.15 \mathrm{~cm}$. were no abnormalities in his gait or posture. We did not observe resting tachypnea or paradoxical diaphragm phenomenon during normal breathing. However, the patient complained of mild shortness of breath while running or climbing stairs. After 2 months, follow-up ultrasonography showed paradoxical movement of the right diaphragm, which indicated complete paralysis (Fig. 3). The patient underwent pulmonary rehabilitation 2 hours daily after the initial diagnosis, including air-stacking and inspiratory muscle training, in order to prevent atelectasis and pneumonia of the right lung.

The informed consent was waived.

\section{DISCUSSION}

The diagnosis of neurosyphilis was based on the laboratory tests, CSF analysis, and ophthalmological tests. Chest X-ray, ultrasonography, and electrophysiological studies were performed to assess the diaphragm paralysis. The present patient denied any history of trauma or respiratory distress, and his previous chest radiographs had been unremarkable. The evidence of phrenic nerve neuropathy from the electrophysiological studies and laboratory tests revealed that the patient's symptoms were likely due to neurosyphilis.

Unilateral diaphragm paralysis due to phrenic nerve palsy is strongly associated with several conditions, such as multiple sclerosis, cervical spinal cord injury, tumor infiltration, motor neuron disease, and idiopathic causes. Previous studies have also reported that herpes zoster virus, pneumonia, and Lyme borreliosis are infectious causes of diaphragm paralysis due to phrenic nerve involvement [8-10]. However, phrenic nerve palsy with viral involvement is very rare, particularly in neurosyphilis. To the best of our knowledge, diaphragmatic paralysis associated with neurosyphilis has rarely been reported [4-6]. Brage [4] reported that hemidiaphragm paralysis was associated with syphilis but that movement of the diaphragm did not recover after penicillin or bismuth treatment, as in our patient. Mindel et al. [7] reported that peripheral neuropathy is a rare clinical manifestation of both primary and secondary syphilis. This report 
highlights an unusual case of neurosyphilis-associated unilateral diaphragm paralysis due to phrenic neuropathy.

Although the present patient experienced unilateral involvement of the phrenic nerve, he may experience bilateral involvement of the nerves as the disease progresses. In such situations, orthopnea can limit daily activities and disturb sleep. Further studies should be conducted in order to understand the progression of phrenic neuropathy in neurosyphilis. The information regarding diaphragmatic involvement in neurosyphilis should be made available to clinicians, and diaphragm paralysis, which can lead to shortness of breath and orthopnea in patients with neurosyphilis, should be excluded.

\section{CONFLICT OF INTEREST}

No potential conflict of interest relevant to this article was reported.

\section{ACKNOWLEDGMENTS}

This work was supported by a 2-year Research Grant of Pusan National University.

\section{AUTHOR CONTRIBUTION}

Conceptualization: Huh S, Ko HY. Methodology: Huh S, Chung JH, Kwon HJ, Ko HY. Formal analysis: Huh S. Funding acquisition: Ko HY. Project administration: Huh S. Visualization: Huh S, Chung JH, Kwon HJ. Writing - original draft: Huh S, Chung JH, Kwon HJ. Writing - review and editing: Huh S, Chung JH, Kwon HJ, Ko HY.
Approval of final manuscript: all authors.

\section{REFERENCES}

1. Rudrappa M, Kokatnur L, Chernyshev O. Neurological respiratory failure. Diseases 2018;6:7.

2. Ratnayake EC, Shivanthan C, Wijesiriwardena BC. Diaphragmatic paralysis: a rare consequence of dengue fever. BMC Infect Dis 2012;12:46.

3. Betensley AD, Jaffery SH, Collins $\mathrm{H}$, Sripathi N, Alabi F. Bilateral diaphragmatic paralysis and related respiratory complications in a patient with West Nile virus infection. Thorax 2004;59:268-9.

4. Brage D. Unilateral paralysis of the phrenic nerve of syphilitic origin. Sem Med 1956;108:939-43.

5. Riley EA. Idiopathic diaphragmatic paralysis; a report of eight cases. Am J Med 1962;32:404-16.

6. Briscoe $\mathrm{C}$. The diagnosis of unilateral phrenic nerve paralysis: an important point in mediastinal localization. Lancet 1925;205:376-81.

7. Mindel A, Tovey SJ, Timmins DJ, Williams P. Primary and secondary syphilis, 20 years' experience. 2 . Clinical features. Genitourin Med 1989;65:1-3.

8. Ricoy J, Rodriguez-Nunez N, Alvarez-Dobano JM, Toubes ME, Riveiro V, Valdes L. Diaphragmatic dysfunction. Pulmonology 2019;25:223-35.

9. Piliero PJ, Estanislao L, Simpson D. Diaphragmatic paralysis due to isolated phrenic neuropathy in an HIV-infected man. Neurology 2004;62:154-5.

10. Faul JL, Ruoss S, Doyle RL, Kao PN. Diaphragmatic paralysis due to Lyme disease. Eur Respir J 1999;13:700-2. 